\title{
Avant-propos au vingtième numéro de Communication \& Organisation
}

\section{Hugues Hotier}

\section{(2) OpenEdition}

1 Journals

Édition électronique

URL : http://journals.openedition.org/communicationorganisation/2545

DOI : 10.4000/communicationorganisation. 2545

ISSN : 1775-3546

Éditeur

Presses universitaires de Bordeaux

\section{Édition imprimée}

Date de publication : 1 novembre 2001

ISSN : 1168-5549

Référence électronique

Hugues Hotier, "Avant-propos au vingtième numéro de Communication \& Organisation »,

Communication et organisation [En ligne], 20 | 2001, mis en ligne le 27 mars 2012, consulté le 21

septembre 2020. URL : http://journals.openedition.org/communicationorganisation/2545; DOI :

https://doi.org/10.4000/communicationorganisation.2545

Ce document a été généré automatiquement le 21 septembre 2020.

(c) Presses universitaires de Bordeaux 


\title{
Avant-propos au vingtième numéro de Communication \& Organisation
}

\author{
Hugues Hotier
}

1 Ce n'est pas sans une certaine émotion et une légitime fierté... Si cet avant-propos débutait ainsi, il aurait un petit air suranné de discours convenu. Et pourtant... L'émotion est bel et bien là. Et la fierté aussi. Pourquoi le cacher ?

2 Il y a juste dix ans, exactement cinq ans après la fondation du GREC/O, nous décidions de créer une revue de recherche. Ne voyez pas là un caprice de chercheur. D'ailleurs, les chercheurs pourraient-ils, pour peu qu'ils en aient l'envie, s'autoriser des caprices? C'était simplement une réponse à un constat : il n'existait aucune revue qui fût dédiée à la communication des organisations. Cette discipline, ou cette sous-discipline des sciences de l'information et de la communication, devait se contenter des rubriques que lui concédaient des revues de management ou de gestion. Nous étions convaincus, en créant Communication \& Organisation, de répondre à un besoin et à une demande. La décision fut prise en novembre 1991 et, coïncidant avec le cinquième anniversaire de la fondation du Groupe de Recherche en Communication des Organisations, marquait un nouveau départ ou, à tout le moins, un changement qualitatif. En créant une revue, nous nous engagions dans ce qu'on appelle la valorisation de la recherche alors que, jusque-là, nous nous cantonnions à la production de travaux de recherche, à notre propre production. Ce fut, effectivement, un tournant dans la vie de notre laboratoire.

Quand je relis mon premier avant-propos, celui du premier numéro, paru en mai 1992, je me dis qu'il pourrait être reproduit presque mot pour mot aujourd'hui. D'ailleurs, hormis des améliorations ponctuelles, essentiellement au niveau de la forme, notre revue a peu changé. Je relèverai trois éléments de ce discours d'ouverture :

4 - "Communication \& Organisation s'adresse aux chercheurs mais aussi aux praticiens auxquels elle propose une réflexion distanciée par rapport à une pratique quotidienne trop souvent vécue dans la hâte et le stress peu propice au recul conceptuel. " Cette orientation ne s'est jamais démentie. Il est d'ailleurs significatif que, depuis quelques années, on a vu naître en plusieurs lieux des associations de professionnels de la communication qui se réunissent pour réfléchir sur leur pratique. Je citerai, à titre d'exemple, 
"Communication et Réflexion » dont le siège est à Bordeaux et qui a des sections à Lyon et à Paris. À ces communicateurs, qu'ils travaillent en agence, en entreprise ou dans une collectivité, notre revue s'offre comme un outil de réflexion.

- «Faut-il préciser que Communication \& Organisation est un lieu de publication et de confrontation et que les articles que vous proposerez seront soumis, anonymement, à un comité de lecture international. » Nous n'avons jamais dérogé à cette règle qui garantit le niveau scientifique d'une revue de recherche. Les articles que nous recevons se répartissent en deux catégories. D'une part, ceux qui sont proposés pour le dossier thématique (170 pages sur les 300 que comporte chaque livraison normale, hors série et numéros spéciaux exclus) qui sont pris en charge par les responsables scientifiques dudit dossier. Ce sont ces responsables qui envoient les articles aux lecteurs choisis en fonction de leur compétence par rapport au sujet. Les deux lecteurs qui reçoivent chaque article émettent un avis qui peut aller de l'acceptation sans réserve au rejet en passant par la proposition de modifications. S'ensuit une navette entre l'auteur, le responsable scientifique du dossier et les lecteurs. Bien entendu, l'auteur ne connaît pas les noms des lecteurs, pas plus que ceux-ci ne connaissent celui de l'auteur. Si nécessaire, l'éventuel litige est examiné et tranché par le comité éditorial. Composé de cinq enseignants-chercheurs membres du GREC/ $/ \mathrm{O}^{1}$, ce comité éditorial traite les articles des autres rubriques selon les principes énoncés précédemment. Dès la création, nous avons voulu échapper à la contrainte des numéros thématiques qui privent beaucoup de revues de la possibilité de publier des articles intéressants mais hors sujet. C'est pourquoi nous avons ouvert la rubrique « Analyses » qui propose des articles de fond, dont le caractère conceptuel est évident, sans être soumis à un calendrier des thèmes. Tout cela suppose une réelle discipline si l'on veut publier en temps et en heure. Ce qui, reconnaissons le, est rarement le cas. Notre point faible, il est vrai, se trouve au niveau de l'impression. Pour des raisons qui relèvent de l'économie, nous avons choisi, sauf exception, de travailler avec l'imprimerie intégrée de l'université à laquelle nous appartenons. Mais, outre que les délais sont rarement tenus, les surprises sont aussi nombreuses qu'insupportables. Ainsi, par exemple, la sortie du $n^{\circ} 19$ a-t-elle été retardée de six semaines parce qu'il a fallu recommencer la couverture trois fois... On aura compris que, soucieux de sortir ce vingtième numéro à une date précise et avec quelque célébration, nous nous soyons adressés à un imprimeur externe. Je voudrais ajouter que deux femmes vivent au jour le jour le stress de la gestation et de la diffusion. Aline Simon-Lalande, secrétaire de la publication, prend la revue en charge depuis la réception des articles (éventuellement après relance...) jusqu'au prêt à clicher. C'est elle qui fait la mise en page et les vérifications typographiques. C'est à elle qu'incombe la difficile relation avec les auteurs qui, de ce point de vue, ne sont pas toujours irréprochables. On sait bien que les intellectuels - je n'échappe pas à ce reproche que je m'adresse aussi à moi-même - éprouvent beaucoup de difficultés quand il s'agit de se soumettre à des impératifs de forme. Surtout quand il faut faire tenir une pensée riche et foisonnante dans un nombre de signes limité! Il faut beaucoup de diplomatie à Aline pour faire respecter les normes tout en apaisant les mouvements d'humeur des auteurs ou... du directeur de la publication. Responsable de la diffusion, Marie Navarro gère les abonnements aussi bien que les commandes, qu'elles émanent de particuliers ou de libraires. Souvent, nous avons évoqué avec elle le recours à un logiciel. Toujours, nous avons dû renoncer devant la dépense. Car les impératifs économiques sont, avec l'importance du travail à fournir, des motifs de renoncement pour beaucoup de centres de recherche qui aimeraient éditer une revue. 
Voulant éviter le mot "archaïque ", je dirai que nos méthodes sont artisanales. Par la force des choses. Marie Navarro et Aline Simon-Lalande sont des collaboratrices précieuses sans qui notre revue aurait beaucoup de mal à exister. Qu'elles soient ici remerciées.

- "International est le dernier mot-clef de cette préface car Communication \& Organisation se veut revue francophone plutôt que française. » Là encore, les engagements ont été tenus. Il n'y a d'ailleurs rien d'étonnant à cela puisque ces engagements étaient, en fait, des principes fondateurs. Nos dossiers ont été indifféremment pris en charge par des universitaires belges, français et québécois. Les articles de nos vingt numéros ont été écrits par des auteurs de ces nationalités d'expression française mais aussi par des Africains et des Chinois écrivant en français. Nous avons même publié des articles d'anglophones, Canadiens et Américains, qui nous les avaient fournis en traduction. En ce sens, notre revue de langue française apporte à la communauté francophone un contenu international précieux.

7 Il manquait une précision à cette profession de foi du premier numéro : l'ouverture aux jeunes chercheurs. Nous ne l'avions pas dit mais nous l'avons fait tant cela constituait pour nous une évidence. Il nous paraît important que les doctorants, par exemple, puissent voisiner avec les chercheurs connus. Ce fut le cas dès le premier numéro où fut publié un article d'une doctorante en fin de thèse à côté de ceux de Jacques Ellul et Robert Escarpit. Là aussi, la pratique relève du principe.

Un dernier mot pour me féliciter que le dossier thématique de ce vingtième numéro, dont on aura compris la valeur symbolique, soit réalisé par deux membres du Groupe de Recherche en Communication des Organisations: Valérie Carayol, maître de conférences à l'Université Michel de Montaigne-Bordeaux 3, et Gino Gramaccia, professeur à l'Université Bordeaux 1.

Et, pour terminer tout à fait, je voudrais dire ma gratitude à tous ceux qui nous ont été fidèles au cours de cette décennie. Exemplaire est la fidélité de David Cessac, ancien étudiant du DESS en Information et Communication des Organisations qui continue à dessiner les illustrations de notre couverture. Ce n'est qu'un exemple parmi des dizaines d'autres mais il est significatif de l'attachement à notre revue et de cette amicale complicité qui lui a permis de naître et d'exister et qui lui permettra, je l'espère, de se perpétuer.

10 Je donne à nos lecteurs rendez-vous dans dix ans !

\section{NOTES}

1. Dominique Blin, Valérie Carayol, Hélène Dufau-Rossi, Béatrice Galinon-Mélénec, Gino Gramaccia 


\section{AUTEUR}

HUGUES HOTIER

Directeur scientifique de Communication \& Organisation et du Groupe de Recherche en Communication des Organisations 